TECHNICAL TRANSACTIONS 10/2018

CZASOPISMO TECHNICZNE 10/2018

COMPUTER SCIENCES

DOI: $10.4467 / 2353737 X C T .18 .146 .9094$ SUBMISSION OF THE FINAL VERSION: 18/09/2018

\author{
Dariusz Żelasko (iD) orcid.org/0000-0001-5125-3647 \\ dzelasko@pk.edu.pl \\ Cracow University of Technology, Faculty of Physics, Mathematics and Computer \\ Science
}

\title{
ENSURING THE QOS IN COMPUTER NETWORKS THROUGH THE USE OF THE PAY\&REQUIRE MULTI-AGENT SYSTEM AND ELECTRONIC AUCTIONS
}

\author{
ZAPEWNIANIE JAKOŚCI USŁUG W SIECIACH KOMPUTEROWYCH POPRZEZ \\ WYKORZYSTANIE SYSTEMU WIELOAGENTOWEGO PAY\&REQUIRE \\ I AUKCJI ELEKTRONICZNYCH
}

\begin{abstract}
In modern computer networks, agent-based systems are widely used. The very fundamental usage of agents are for management systems e.g. supporting SNMP-based databases and multiple client-server communications. This paper describes the Pay\&Require mechanism implementing a multi-agent approach for a QoS-enabled computer network. The unique features of the P\&R system are its comprehensive approach to the cooperation of agents dedicated to traffic monitoring, the selective response to the QoSoriented admission of user requests, event-driven reconfiguration and transmission quality brokerage. The brokerage in quality purchase was based on different models - from a simple reactive agent to an agent with learning capabilities. The presented approach uses auctions in which agents (brokers) can buy quality parameters according to customer requirements. The use of auctions allows the dynamic pricing of data transmission services.
\end{abstract}

Keywords: agent negotiations, market-based price modelling, auctions, computer networks, quality assurance, QoS, Pay\&Require

\section{Streszczenie}

We wspólczesnych sieciach komputerowych bardzo często wykorzystywane są systemy agentowe. Podstawowym zastosowanie agentów jest zarządzanie systemami, np. SNMP i komunikacja klient-serwer. W artykule opisano mechanizm Pay\&Require w którym zastosowano podejście wieloagentowe do zapewniania QoS w sieciach komputerowych. Cechą wyróżniającą mechanizm P\&R jest kompleksowe podejście do współpracy agentów odpowiedzialnych za monitorowanie ruchu, reakcja na żądania użytkowników dotyczące QoS, rekonfiguracja na podstawie zdarzeń i pośrednictwo w zakupie jakości. Pośrednictwo to opiera się na różnych modelach - od prostego agenta reaktywnego do agenta z możliwością uczenia. Prezentowane podejście wykorzystuje aukcje, w których agenci (pośrednicy) mogą kupować parametry dotyczące jakości zgodnie z oczekiwaniami klienta. Wykorzystanie aukcji pozwala na dynamiczne kształtowanie cen usług transmisji.

Słowa kluczowe: negocjacje, metody rynkowe, aukcje, sieci komputerowe, zapewnianie jakości, QoS, Pay\&Require 


\section{Introduction}

The continuous development of both hardware (such as computing power, memory size and speed) and software can be observed in modern computer networks. More and more, techniques derived from IT solutions such as agent systems are found. The use of agent systems aims to improve the way in which software is designed and implemented. The design of the system using elements that are useful from the point of view of the development of modern software such as reactivity, proactivity and social behaviour, is characteristic of the agent-based approach $[1,2]$. Reactivity is the capability to monitor the environment in which the agent is located and to respond to changes. Proactivity is the demonstration of initiative and goal-oriented behaviour. Social behaviour, in turn, means that the agent should interact with other agents in the environment. Another important issue is the agent's independence - it should be able to operate without external interference. A single agent is perceived as an intermediary, usually between the user and the environment in which it operates.

The classical approach in networks is to use agents in management systems (management agents) $[7,12,13]$. The task of such an agent is to provide the interface that enables management of the device while taking into account the received feedback and the implementation of the reconfiguration processes [11]. The operation of computer networks is based on traffic management, which consists of traffic stream classification, QoS qualitative analysis, route definition, and the definition and implementation of active traffic management methods in nodes.

Classical IP networks do not guarantee transmission quality - rather, they deliver a 'best effort' transmission service. IP networks with IntServ (not used due to the lack of scalability) or DiffServ [8] (providing great capabilities despite being used for more than 20 years) provide another step in the development of QoS traffic management. Techniques for traffic streaming in a connection mode are ATM (asynchronous transfer mode), MPLS (multiprotocol label switching) [9], GMPLS (generalised MPLS), or the latest SDN (software-defined networking) [10] concept provide QoS guarantees through centralised asset monitoring and the defining of control parameters. A multi-agent system, the primary role of which is to provide updates and distribution of control rules, can be used in each of these concepts. Using agents for network control and quality assurance is a non-classic and innovative approach.

The Pay\&Require concept shown in this paper is an innovative approach to quality assurance in computer networks [15]. An agent-based approach to network management was used to achieve decentralisation of the solution. Electronic auctions play an important role in decision-making in the agent-based network management system. It is important to define the rules for the settlement of auctions and the value paid for acquiring the goods. We can distinguish two approaches - first-price and second-price [6]. In first-price auctions, the participant who made the best bid wins and receives the auction item for the bid amount. In second-price auctions, the client bidding the most wins, but pays as much as the next auction participant offered. Auctions can also be classified according to the openness of information and we can distinguish between open-cry and sealed-bid auctions. In open-cry auctions, the 
participants have full knowledge of the actions of other auction participants. The participants do not have access to information about the activities of other participants in sealed-bid auctions. For auctions where one good is offered, the following different auction protocols are defined. The simplest protocols are first-price-sealed-bids, where the auction is held in one round and the participant who makes the highest bid wins. As the auction is held in one round, it is very important that users bid according to the maximum accepted amount from the very beginning. Another protocol is the second-price-sealed-bid, and in this case, the participant who made the highest bid wins but pays the second highest amount.

Auctions for multiple goods are more complex because many factors can affect their course. Participants can apply using several different bidding methods. The first of these is the XOR method. It consists of placing many different bids, and the user will only pay according to one of them. In situations where it is possible to meet several conditions at the same time, the user pays the highest amount of the individual bids. For example, when $d 1, d 2, d 3, d 4$ goods are available, the user can submit the bid according to the assumptions shown in equation (1).

$$
O f=\left(\left\{d_{3}, d_{4}\right\}, 20\right) \operatorname{XOR}\left(\left\{d_{1}\right\}, 10\right)
$$

Such a notation means that the user can pay 20 if the user receives goods $d 3$ and $d 4$; the user pays 10 when receives item $d 1$ only; but pays 20 when they receive goods $d 1, d 3, d 4$. Another possibility is the so-called OR bids, in which the final bid is the sum of the component bids. For the example shown in equation (1) this means that the participant pays 20 when receiving goods $d 3$ and $d 4,10$ when receiving item $d 1$ only, and 30 when receiving goods $d 1$, $d 3, d 4$.

\section{Pay\&require concept}

the Pay\&Require (P\&R) concept is an alternative solution to quality assurance techniques in computer networks. Its main assumption is that data transmission takes place at the guaranteed quality level. Quality is defined by parameters such as throughput, transmission delay and number of lost packages. Obtaining the appropriate transmission quality is achieved by selecting such routing paths that the parameters meet the client's requirements. Quality is monitored at specified intervals to determine if the transmission quality has changed. This is achieved through active measurements performed by test transmission on each network link. This determines the current quality of each link. When quality satisfies client requirements, transmission takes place using the established paths. However, the network reconfigures if a deviation from the required parameters is found and this results in the changing of routing tables. Such a solution enables transmitting the data of different clients via different paths.

The concept separates the physical layer (network devices) from the management-control layer (Fig. 1). The devices responsible for the packet routing $\left(R_{x}\right)$ operate in the physical layer. Agents responsible for the monitoring of transmission parameters and reconfiguring the network $\left(A g_{x}\right)$ operate in the control layer. Monitoring agents periodically check the current 
quality. When parameters change, agents send this information to the agents responsible for reconfiguration.

This type of agent defines new paths and then performs the reconfiguration of physical layer devices based on quality and the client's requirements. Reconfiguration of this layer consists of the transmission of configuration commands which allow devices to route packages based on new rules. Agents responsible for the purchase of quality (market) comprise a separate layer. They are intermediaries between the client $\left(\mathrm{Ca}_{x}\right)$ and the environment where the auctions or negotiations are conducted. Another agent represents the service provider $(\mathrm{Oa})$.

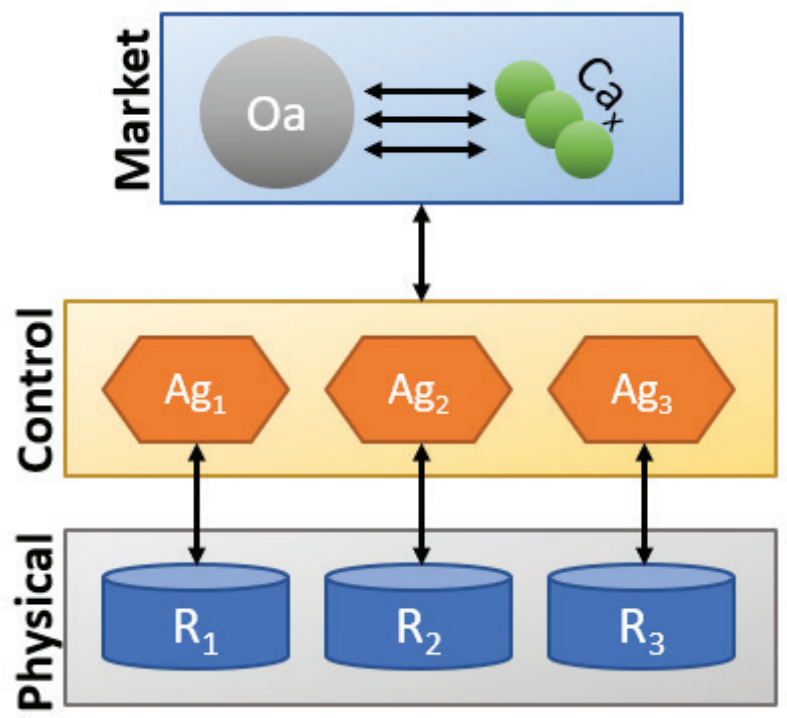

Fig. 1. Pay\&Require layers

\section{Architecture of agents}

Among the numerous types of agents based on different architectures and with different algorithms $[1,3,4]$, cognitive and reactive agents were used in this research. The simplest types of agents, the task of which is to mediate in operation without the developed learning and decision-making skills, are often used in computer networks. The P\&R concept uses several types of agents.

\subsection{Monitoring agent}

The first agent is responsible for monitoring the transmission quality (the so-called monitoring agent). This agent belongs to the group of reactive agents, whose operation is based on the mapping of the situation in the environment to a particular action. There are one or more instances of an agent of this type within each active device on the network. The agent can monitor the entire device or its interfaces - in this case, multiple monitoring agents can operate as a part of one device. Each agent of this type is responsible for monitoring 
the parameters of individual links. The agent can make decisions based on predefined rules or based on its own experience acquired during the operation. Qualitative parameters may be subject to a minimum change that does not noticeably affect the quality of the service. Therefore, the agent should act on the basis of parameter value ranges and should be able to learn the correct behaviour during its life.

\subsection{Route reconfiguration agent}

The second agent used in the P\&R concept is responsible for the reconfiguration (the so-called reconfiguration agent); it is an example of a reactive agent. Once the monitoring agent has determined that the parameters excessively differ from the assumed values, it reports the need to change the route. The route reconfiguration agent starts the process of assigning new paths; a path is created between users based on both the quality information for each link on the network and the client information. The path must satisfy client requirements - the quality cannot be worse and should not be better. Subsequently, after assigning new paths, the agent will send the new configuration to a subordinate device. An agent can monitor one device or a group of devices.

The monitoring agent and the route reconfiguration agent may be discrete elements or be implemented as a single agent with both functionalities. Figure 2 shows the $A g$ agent model along with details of the communication between agents of the same type and a router (physical layer). The first step is the exchange of information regarding networks directly connected to the agent-supervised router. Subsequently, agents send the information regarding networks they have learned from their neighbours to one another. The exchange of messages ends when all agents have information about the network topology. Agents that monitor devices to which clients are connected have information about the quality required by each client. The transmission path is then selected which will be used for a given client. Agents configure the router(s) they monitor based on the received information.

The agent periodically verifies the parameters of the device links that it monitors. The test consists of sending a test stream of information through each link. Parameters such as throughput,

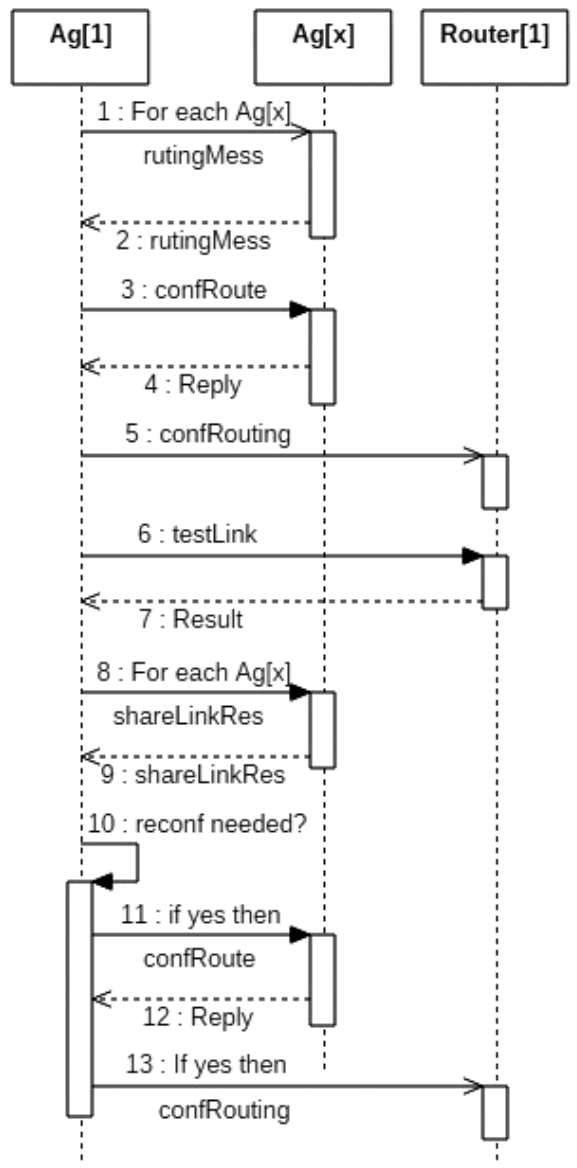

Fig. 2. Agent $A g$ operation 
transmission time, delay, and the number of lost packages are determined. Information about measured parameters is sent to neighbouring devices. The agent evaluates the paths used for transmission by clients based on the received information. The routes are reconfigured when the parameters deviate from the assumptions.

\subsection{Agent-trader}

Agent-trader is the last type of agent. This agent is responsible for the quality trade such a type of agent may represent the provider or the client. In the simplest case, the user pays a certain amount for quality - this payment can be made for a specified interval (e.g. one month). This is not the only solution since it may happen that the user needs quality only for the duration of a specific transmission or for a particular service. The system then enables the user to temporarily change the quality. In the simplest terms, the agent system will act as an intermediary on the purchase. We can introduce various methods of market purchasing and negotiating here. Depending on the strategy of the agent, this can be classified as reactive the simplest case being mapping of the situation to the decision. However, intelligent agents are used in more complex strategies where an agent uses historical data and other knowledge.

Each quality parameter can be a separate item - then the negotiations involve many goods at the same time, the combination of which will satisfy the client. It is also possible to translate a combination of qualitative parameters using a certain scale.

The auction mechanism can be used with regard to the allocation of goods. This allocation type is used when more than one party is interested in goods. The purpose of the auction is the allocation of goods to one participant. Each auction participant has an assigned strategy and the maximum amount that the agent is willing to pay for a given transmission quality (being its evaluation of the value of a given quality of service) is an important element of the strategy. The participants should not disclose their strategies. Auctions run according to certain rules - the auction protocol.

In the P\&R concept, the agent representing the auction participant is not solely an intermediary but can make autonomous decisions based on the adopted strategy and the amount at its disposal. The agent can act carefully, either bidding only small amounts or bidding high amounts. Amounts may be fixed, random within a certain range or may result from the experience of the agent during previous auctions. Several agent action strategies have been adopted:

1. The agent has information about the quality sought by the client and the maximum amount that the client can pay. The bid $(O f)$ is only made to a specified maximum. Equation (2) shows the method of bidding for this strategy, where $q$ is quality and $\max (c 1(q))$ is the maximum amount the client $i$ can assign for the purchase of quality $q$.

$$
O f(q) \leq \max \left(c_{1}(q)\right)
$$

2. The agent has information about the maximum amount, expected quality and the possibility of deviation from quality within a certain range. This situation can be beneficial to the client, who wants transmission at an optimum price while maintaining 
an acceptable level of quality. Equation (3) shows the bidding method. Qm is the margin of deviation from the assumed quality which is acceptable to the client.

$$
O f(q \pm Q m) \leq \max \left(c_{1}(q+Q m)\right)
$$

3. Where the same quality is offered successively in several different auctions (the provider can assure a certain level to several clients simultaneously), and the agent has information about the number of such auctions, it is possible to adopt a strategy where the user watches the results of subsequent games. In the last round, the agent takes part in the game by predicting its outcome based on previous games. Information received by the agent may be partial (final result of the auction only) or full (final result and bids submitted by each agent). In the case of partial knowledge, the agent can make predictions based solely on the results of previous games. Therefore, it may assume a certain probability that it will win the auction if it places a certain bid. There is a more complex model in which the agent has full information on the course of all games and bids submitted by other participants. With this approach, the agent can learn the behaviour of other players and learn about their strategies. A behaviour model can be created for each competitor on this basis. This model is used at the stage of submitting an offer such that the agent wins the auction with a certain probability while paying the optimum amount (not overpaying). The agent with knowledge of competitors can compare their models and then assume a strategy that will allow it to win. Whether or not an agent can have full or just partial information about previous auctions depends on the system configuration and the adopted auction protocol.

\section{Findings}

The research was conducted for both single-item auctions and combinatorial (multiple goods) auctions. In the case of single-item auctions, it is necessary to transform quality (resulting from many parameters) based on a certain mapping of parameters to transmission quality. With such a solution, a problem which affects many goods (e.g. throughput, transmission delay, package loss rate), can be reduced to a simpler decision-making problem.

\subsection{One-time auctions of single items}

The research was conducted for an agent whose job is to make a purchase for the client. The quality of data transmission service is defined within the range of 0 to 5 . The research was conducted for both first-price and second-price auctions. Auctions are carried out in one round, i.e., agents bid simultaneously and only once. In both cases, the highest bid wins, but the final amount is the second largest of the submitted bids in the second-price auction. Ten clients were defined for the simulation. Client number 1 is analysed for the result of the game. Other clients are its opponents, but they do not exchange information about the 
adopted strategy. For strategy 1 , all clients are interested in the same quality. Table 1 shows the maximum amounts accepted by clients.

Table 1. Opponents for strategy 1

\begin{tabular}{|c|c|c|}
\hline Client & Amount & Quality \\
\hline K2 & 4 & 3 \\
\hline K3 & 4 & 3 \\
\hline K4 & 4 & 3 \\
\hline K5 & 14 & 3 \\
\hline K6 & 14 & 3 \\
\hline K7 & 14 & 3 \\
\hline K8 & 24 & 3 \\
\hline K9 & 24 & 3 \\
\hline K10 & 24 & 3 \\
\hline
\end{tabular}

Figure 3 shows the results obtained for strategy 1 . The measurement was performed for three different maximum amounts acceptable for client $1-10,20$, and 30 (measures 1, 2, and 3 , respectively). The chart shows two values - the bid value, which is the amount the client 1 has offered, and the winning value, which is the winning amount. When the value is greater than or equal to the winning value, it means that the agent representing client 1 has won. Taking into account the strategies of other players, agent 1 wins only if its maximum is greater than the maximum of other players. This situation is based on the assumption that each agent will bid the maximum amount.

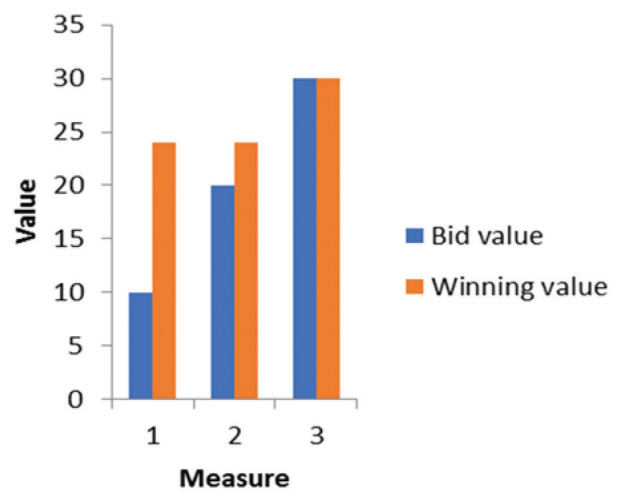

Fig. 3. Results for first-price strategy 1

For strategy 2, it is assumed that in addition to the maximum amounts, the clients are also interested in a specific quality that does not have to be the same to them. Client 1 , for whom the outcome of the game is considered for strategy 2 , has a defined quality acceptance threshold of \pm 1 . Table 2 shows the adopted strategies for these measurements. 
Table 2. Opponents for strategy 2

\begin{tabular}{|c|c|c|}
\hline Client & Amount & Quality \\
\hline K2 & 4 & 1 \\
\hline K3 & 4 & 2 \\
\hline K5 & 14 & 2 \\
\hline K4 & 4 & 3 \\
\hline K6 & 14 & 3 \\
\hline K8 & 24 & 3 \\
\hline K7 & 14 & 4 \\
\hline K9 & 24 & 4 \\
\hline K10 & 24 & 5 \\
\hline
\end{tabular}

Figure 4 shows the results for strategy 2. Also in this case, three measurements were made according to the maximum amounts accepted by client 1 . The situation was analysed depending on the expected quality $(3 \pm 1)$ for each measurement. When the maximum amount is 10 , the agent does not win the auction despite the quality acceptance threshold. In turn, when the maximum amount is 20 , the agent wins the auction for a quality level of 2 and lose it for a different amount. When the maximum amount of 30 is accepted, the agents win for each quality and pay that amount. Therefore, the agent should try to get the best possible quality by playing the high amount.

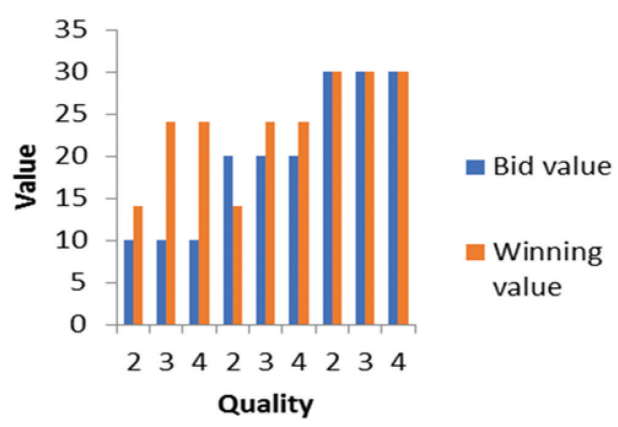

Fig. 4. Results for first-price strategy 2

\subsection{Multiple auctions of single items}

For strategy 3 and for a partial knowledge of the course of previous auctions, it is assumed that the purchase is possible in 10 rounds, where agent 1 watches the results for 9 rounds and then plays the amount in round 10 that is higher than the obtained average but lower than the maximum set by the client (variant 1 ) or such an amount that would enable it to win $90 \%$ of the previous auctions (variant 2).

$$
\frac{1}{2} * \sum_{i=1}^{n} x_{i}<O f(q) \leq \max \left(c_{1}(q)\right)
$$


Equation (4) shows the mode of operation of agent 1 when using variant 1 . The bid depends on quality - $O f(q)$, and it is greater than the calculated average and less than or equal to the maximum acceptable to the client for a given quality.

Figure 5 shows the course of each round along with the average value observed by the agent after 9 rounds and the value estimation through which the agent would win $90 \%$ of the auctions. For a strategy where the agent plays a value of no less than the average of the previous games, one can note that for the strategies adopted by the remaining players, when agent 1 will play at least the amount equal to the average value in round 10, then it will win this round. This is due to the fact that the average was 23.89, and the maximum bid in round 10 was 22 . However, agent 1 is not sure if its bid will win because the strategies of the remaining players may change in successive rounds. Therefore, another option is to submit a bid so that agent 1 would win $90 \%$ of the auctions. According to this approach, agent 1 would play the amount of 26 . Assuming the well-known course of round 10 (the result of 22), agent 1 would win it by bidding 26 , but it would pay more than in the strategy based on the average value. However, if the result of round 10 was greater than the average value, the agent would win by playing the value from variant 2 .

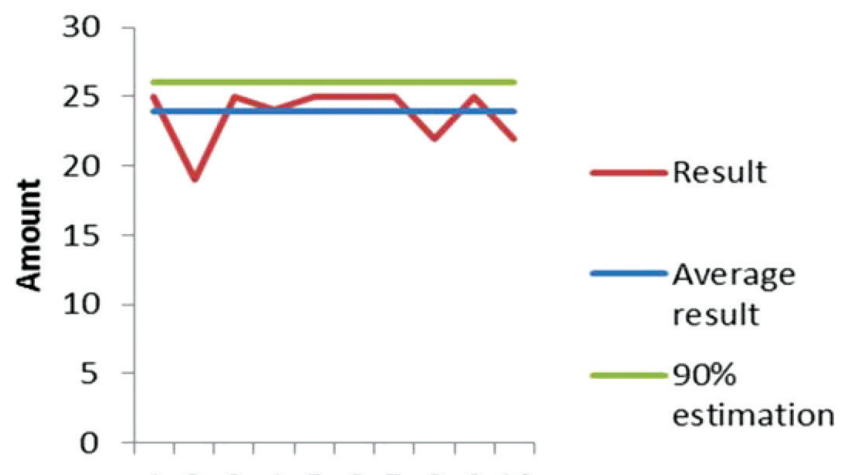

$\begin{array}{lllllllllll}1 & 2 & 3 & 4 & 5 & 6 & 7 & 8 & 9 & 10\end{array}$

Round

Fig. 5. Results for first-price strategy 3 - partial information

For strategy 3, assuming that the agent has full knowledge of all the auctions and bids submitted by competitors so far, it was assumed that all agents took part in 9 rounds. Agents 2, 5, 7, and 10 also take part in round 10 along with agent 1 . With full knowledge of the submitted bids, agent 1 can determine with a certain probability what bid will be placed by each agent in the next round. Figure 6 shows bids submitted by agents in successive rounds and the result of each auction.

Based on 9 rounds, one can note that agent 10 submitted a maximum bid of 25 and a minimum of 6 , agent 2 submitted a maximum of 23 and a minimum of 7 , agent 5 submitted a maximum of 24 and a minimum of 6 , agent 7 submitted a maximum of 25 and a minimum of 7. It can be assumed on this basis that the maximum values of submitted bids are very likely to be the thresholds for each agent. 


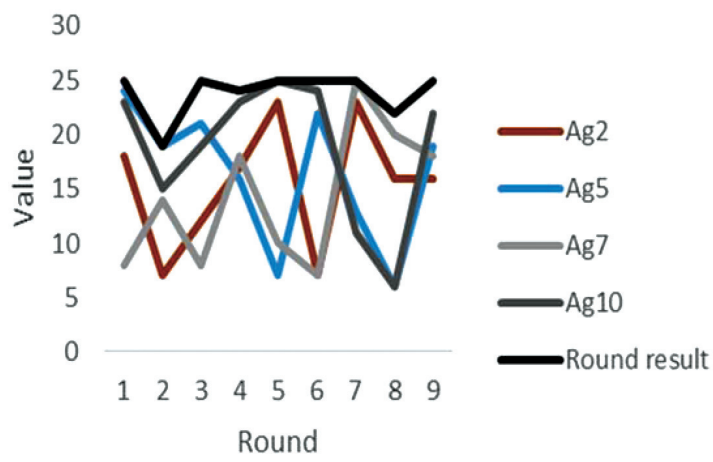

Fig. 6. Results for first-price strategy 3 - full information

Consequently, if agent 1 wants to win round 10, it should submit a bid of no less than the maximum of maximum bids of each competitor. In this case, the maximum is 25 and therefore agent 1 should bid higher than this value, but it should not be much higher in order to not overpay. In this way, agent 1 can learn the behaviour of other players and make a decision to get the expected result based on this knowledge. The more auctions the agent is watching, the more accurate its knowledge will.

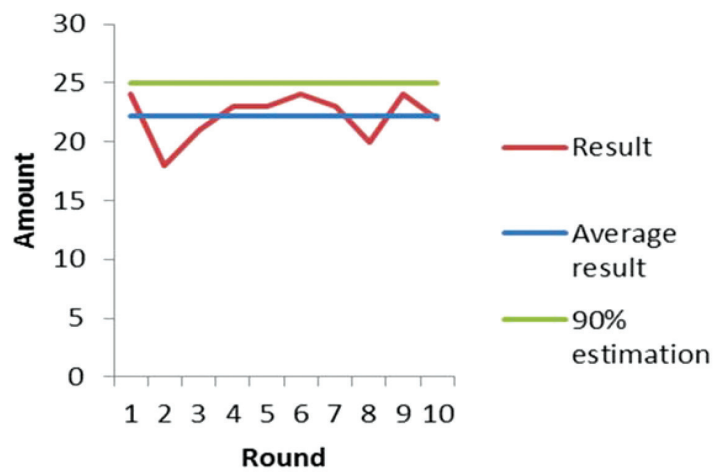

Fig. 7. Results for second-price strategy 3

\subsection{Second price auctions}

A number of experiments have been conducted with regard to the strategies discussed for second price auctions. The average result of 9 games is 22.22 for strategy 3 , having a partial knowledge of the results of previous auctions agent 1 should bid no less than this value in round 10. It will pay the second largest amount; therefore, it should possibly play high. The second largest result in round 10 is 22 , thus no matter how much higher client 1 plays, it will pay 22. Figure 7 shows the results for this strategy and assumptions. For variant 2 , the agent should play no less than 25 to win in $90 \%$ of auctions. This is a minimum value, because it is not known exactly what bids other agents have submitted in the rounds - only the final amount, which is the second largest, is known. Therefore, agent 1 should play as high as possible and most likely will not pay more than 25 in the round where it will participate. 


\subsection{Combinatorial auctions}

Auctions of this type apply to more than one item at a time. In the case of a data transmission service, there are quality parameters such as throughput, transmission delay, and the number of lost packages. Each of these parameters is understood as a separate item and their combination is the basis for the bid. Equation (5) shows the general rule for combinatorial (quality) auctions using logical operations. $O p$ is the logical operator $-X O R$ or $O R$ for the purpose of study, $q_{n}$ is the qualitative parameter, and $a m t_{m}$ is the bid for a given combination of qualities.

$$
O f=\left(\left\{q_{1}, q_{2}, \ldots, q_{n}\right\}, a m t_{1}\right) O P \ldots O P\left(\left\{q_{1}, q_{2}, \ldots, q_{n}\right\}, a m t_{m}\right)
$$

In the study, the $q_{1}$ parameter is throughput, $q_{2}$ is the transmission delay, and $q_{3}$ is the packet loss factor. Client 1 can pay 15 when the parameters are $\{50 \mathrm{Mb} / \mathrm{s}, 4 \mathrm{~ms}\}, 25$ for $\{100 \mathrm{Mb} / \mathrm{s}$, $2 \mathrm{~ms}, 0 \%\}$ and 10 when only a throughput of $50 \mathrm{Mb} / \mathrm{s}$ is guaranteed. Equation (6) shows this strategy. The operator may be $O R$ or XOR for the assumptions. In the case of $O R$ operator, this strategy does not make much sense because it would mean that the user would pay 50 after receiving all parameters at the same time. If the user receives parameters $\{100,2,0\}$, it makes no sense to pay for worse parameters. Therefore, it is appropriate to use the XOR operator. In such a situation, the user pays the maximum amount if the user's requirements are met.

$$
O f=(\{50,4\}, 15) O P(\{100,2,0\}, 25) O P(\{50\}, 10)
$$

Unfortunately, it is hard to ensure a constant level of quality parameters. There are always changes that may not have a significant impact on the final quality. Consequently, an acceptance threshold should be defined for each parameter. This means that parameters within a given range are still acceptable to the client. In the simplest case, the client defines the acceptance threshold for each parameter. In a more complex case, it is possible to define the acceptance ranges for each amount separately. Another possible solution is to define whether the individual parameter values are minimum or maximum values. One can assume for the above example that the client specifies the minimum throughput, maximum delay, and the maximum package loss rate.

\section{Conclusions}

Agent systems in computer networks are still innovative concepts. They can be used both for monitoring and reconfiguring the network and to represent and act on behalf of the client, e.g. when purchasing quality of data transmission services. Purchases can be conducted using a variety of protocols and methods - one can define different strategies for the participation of agents in auctions on their basis of these protocols and methods. The most important assumption is maximising the profit, i.e. purchasing a certain quality for an effectively 
calculated price. Strategies can work on the principle of a finite automaton (the simplest solution) or with learning elements based on the results of previous games. The results show that it is possible to use agents as intermediaries between clients and the provider.

The Pay\&Require concept is a complex mechanism used for quality assurance in the computer network and dynamic pricing. The client pays for the quality that is guaranteed by monitoring the quality parameters and reconfiguration of transmission paths as needed specific types of agents are responsible for monitoring and reconfiguring the network. In turn, the client can purchase quality by participating in the auction in which it is represented by an agent. This solution allows the entire network to be dynamically managed, which improves its operation.

\section{References}

[1] Wooldridge M., An Introduction to MultiAgent Systems, John Wiley \& Sons Ltd, 2002.

[2] Franklin S., Graesser A., Is it an agent, or just a program?: A taxonomy for autonomous agents, International Workshop on Agent Theories, Architectures, and Languages, 1996.

[3] Weiss G., Multiagent systems, Massachusetts Institute of Technology, 2013.

[4] Russell S., Norvig P., Artificial Intelligence A Modern Approach, Pearson Education Inc., 2010.

[5] Fatima S., Kraus S., Wooldridge M., Principles of automated negotiation, Cambridge University Press, 2015.

[6] Wellman M. P., Trading agents, Morgan \& Claypool, 2011.

[7] Simple Network Management Protocol (SNMP) Applications (RFC3413).

[8] New Terminology and Clarifications for Diffserv (RFC3260).

[9] Multiprotocol Label Switching Architecture (RFC3031).

[10] Software-Defined Networking (SDN): Layers and Architecture Terminology (RFC7426).

[11] Mitrović D., Budimac Z., Ivanović M., Vidaković M., Improving fault-tolerance of distributed multi-agentsystems with mobile network-management agents, Proceedings of the International Multiconference on Computer Science and Information Technology, 2010.

[12] Bogdanovic D., Autonomic agent for transport networks, Optical Fiber Communications Conference and Exhibition (OFC), 2015.

[13] Olfati-Saber R., Fax J.A., Murray R.M., Consensus and Cooperation in Networked MultiAgent Systems, Proceedings of the IEEE, Vol. 95, issue 1, 2007.

[14] Cetnarowicz K., Paradygmat agentowy w informatyce. Koncepcje, podstawy izastosowania, Akademicka Oficyna Wydawnicza EXIT, Warszawa 2012.

[15] Żelasko D., Cetnarowicz K., Wajda K., Koźlak J., Payઐ Require as concept of variable cost routing in dynamically reconfigured networks, Technical Transactions, 1NP/2016. 